\title{
MINIMAL COCKCROFT SUBGROUPS
}

\author{
by JENS HARLANDER
}

(Received 20 July, 1992)

Statement of results. Consider any group $G$. A $[G, 2]$-complex is a connected 2-dimensional CW-complex with fundamental group $G$. If $X$ is a [G,2]-complex and $L$ is a subgroup of $G$, let $X_{L}$ denote the covering complex of $X$ corresponding to the subgroup $L$. We say that a $[G, 2]$-complex is $L$-Cockcroft if the Hurewicz map $h_{L}: \pi_{2}(X) \rightarrow H_{2}\left(X_{L}\right)$ is trivial. In case $L=G$ we call $X$ Cockcroft. There are interesting classes of 2-complexes that have the Cockcroft property. A [G,2]-complex $X$ is aspherical if $\pi_{2}(X)=0$. It was observed in [4] that a subcomplex of an aspherical 2-complex is Cockcroft. The Cockcroft property is of interest to group theorists as well. Let $X$ be a $[G, 2]$-complex modelled on a presentation $\langle S ; R\rangle$ of the group $G$. If it can be shown that $X$ is Cockcroft, then it follows from Hopf's theorem (see [2, p. 31]) that $H_{2}(G)$ is isomorphic to $\mathrm{H}_{2}(X)$. In particular $\mathrm{H}_{2}(G)$ is free abelian. For a survey on the Cockcroft property see Dyer [5]. A collection $\left\{G_{\alpha}: \alpha \in \Omega\right\}$ of subgroups of a group $G$ that is totally ordered by inclusion is called a chain of subgroups of $G$. Defining $\beta \leq \alpha$ if and only if $G_{\alpha} \leq G_{\beta}$ makes $\Omega$ into a totally ordered set. The main result of this paper is the following theorem.

TheOrem 1. Let $\left\{G_{\alpha}: \alpha \in \Omega\right\}$ be a chain of subgroups of a group $G$. A [G, 2]-complex $X$ that is $G_{\alpha}$-Cockcroft for all $\alpha \in \Omega$ is also $\left(\bigcap_{\alpha \in \Omega} G_{\alpha}\right)$-Cockcroft.

Theorem 1 together with Zorn's lemma give the next result.

Corollary 1. Let $X$ be a Cockcroft $[G, 2]$-complex. Then $G$ contains a minimal subgroup $L$ such that $X$ is L-Cockcroft.

It is a longstanding open question raised by J. H. C. Whitehead [9] whether a subcomplex of an aspherical complex is aspherical. Suppose $X$ is a subcomplex of an aspherical 2-complex $Y$ and denote by $K$ the kernel of the map $\pi_{1}(X)-\pi_{1}(Y)$ induced by inclusion. J. F. Adams [1] showed that if $X$ is not aspherical then $K$ contains a nontrivial perfect subgroup. He studied a certain system of coverings $\left\{X_{K_{\alpha}}\right\}_{\alpha \in \Omega}$ of $X_{K}$, where $\left\{K_{\alpha}\right\}_{\alpha \in \Omega}$ is the set of characteristic subgroups of $K$ such that the quotients $K / K_{\alpha}$ are $C$-conservative for any abelian group $C$. A group $G$ is $C$-conservative if the functor $C \otimes_{C G}$-detects monomorphisms between projective $C G$-modules; i.e. if $\Psi: P \rightarrow Q$ is a homomorphism between projective $C G$-modules and $C \otimes_{C G} \Psi: C \otimes_{C G} P \rightarrow C \otimes_{C G} Q$ is injective, then $\Psi$ is injective (see also Howie [8]). Adams observed that $N$, the intersection of all groups $K_{\alpha}$, is perfect and that $H_{2}\left(X_{N}\right)=0$. If one assumes $X$ to be non-aspherical, then the second homology of the universal covering of $X$ is non-trivial. Thus $X_{N}$ is different from the universal covering and therefore $N$ is non-trivial (see also Howie [6] and [7]).

The proof of Theorem 1 relies on a lemma that deals with arbitrary systems of coverings $\left\{X_{G_{\alpha}}\right\}_{\alpha \in \Omega}$ of a $[G, 2]$-complex $X$. We show that $H_{2}\left(X_{N}\right)$ embeds in $\underset{\leftarrow}{\lim } H_{2}\left(X_{G_{\alpha}}\right)$, where $N$ is the intersection of all the $G_{\alpha}$. We use this result also to characterize non-asphericity of a 2-complex $X$ with $\mathrm{H}_{2}(X)=0$ by the existence of a certain minimal subgroup of $\pi_{1}(X)$. 
THEOREM 2. Let $X$ be a $[G, 2]$-complex with $H_{2}(X)=0$. The following statements are equivalent:

(i) $X$ is non-aspherical;

(ii) there exists a non-trivial minimal subgroup $L$ of $G$ such that $H_{2}\left(X_{L}\right)=0$. Furthermore, if $X$ is non-aspherical, then no group $L$ as in (ii) can have a nontrivial $\mathbb{Z}$-conservative quotient; in particular $L_{a b}$ is torsion.

Assume now that $X$ is a subcomplex of an aspherical 2-complex $Y$. As before let $K$ denote the kernel of the homomorphism $\pi_{1}(X) \rightarrow \pi_{1}(Y)$ induced by the inclusion map. The covering complex $X_{K}$ of $X$ can be viewed as a subcomplex of the universal covering complex $\tilde{Y}$ of $Y$. Since $X_{K}$ and $\bar{Y}$ are 2-complexes, the map $H_{2}\left(X_{K}\right) \rightarrow H_{2}(\tilde{Y})$ induced by inclusion is injective. Since $H_{2}(\tilde{Y})=\pi_{2}(\tilde{Y})=0$ it follows that $H_{2}\left(X_{K}\right)=0$. Theorem 2 applied to the complex $X_{K}$ together with the fact that $X$ is non-aspherical if and only if $X_{K}$ is non-aspherical, yield the following result.

Corollary 2. Let $X$ be a $[G, 2]$-complex that is a subcomplex of an aspherical 2-complex $Y$. Let $K$ be the kernel of the homomorphism $\pi_{1}(X) \rightarrow \pi_{1}(Y)$ induced by inclusion. The following statements are equivalent:

(i) $X$ is non-aspherical;

(ii) there exists a nontrivial minimal subgroup $L$ of $K$ such that $H_{2}\left(X_{L}\right)=0$.

Furthermore, if $X$ is non-aspherical, then no group $L$ as in (ii) can have a non-trivial $\mathbb{Z}$-conservative quotient; in particular $L_{a b}$ is torsion.

I am grateful to Mike Dyer for many helpful suggestions.

Proof of results. Let $X$ be a $[G, 2]$-complex and let $\left\{G_{\alpha}: \alpha \in \Omega\right\}$ be a chain of subgroups of $G$. Denote by $\tilde{X}$ the universal covering complex of $X$ and by $p$ the covering projection

$$
p: \tilde{X} \rightarrow X .
$$

The preimage $p^{-1}(c)$ of each open cell $c$ in $X$ consists of open cells $\tilde{c}_{g}, g \in G$, such that

$$
\left.p\right|_{\bar{c}_{g}}: \bar{c}_{g} \rightarrow c
$$

is a homeomorphism. For each $G_{\alpha}$, the orbit complex $\tilde{X} / G_{\alpha}$, denoted by $X_{\alpha}$, is the covering complex $X_{G_{\alpha}}$ with covering projection

$$
p_{\alpha}: \tilde{X} \rightarrow X_{\alpha} .
$$

Denote by $N$ the intersection $\bigcap_{\alpha \in \Omega} G_{\alpha}$ and by $p_{N}$ the covering projection

$$
p_{N}: \tilde{X} \rightarrow X_{N}
$$

Let $p_{\alpha N}$ be the covering projection

$$
p_{\alpha N}: X_{N} \rightarrow X_{\alpha}
$$

and let $p_{\beta \alpha}$ be the covering projection

$$
p_{\beta \alpha}: X_{\alpha} \rightarrow X_{\beta}
$$

for $\alpha \geq \beta$. The cells in $X_{N}$ and in $X_{\alpha}$ are just $N$ and $G_{\alpha}$ orbits of cells in $\tilde{X}$. So if $N * \tilde{c}=\{n * \tilde{c}: n \in N, \tilde{c}$ an open cell of $\tilde{X}\}$ is an open cell of $X_{N}$, then $p_{\alpha N}$ sends this open 
cell homeomorphically onto the open cell $G_{\alpha} * \tilde{c}$ of $X_{G_{\alpha}}$ and $p_{\beta \alpha}$ sends the open cell $G_{\alpha} * \tilde{c}$ of $X_{\alpha}$ homeomorphically onto the open cell $G_{\beta} * \tilde{c}$ of $X_{\beta}$ for $\alpha \geq \beta$. Now $\left(C_{2}\left(X_{\alpha}\right), p_{\alpha \beta}\right)_{\alpha, \beta \in \Omega}$ is an inverse system of Abelian groups with inverse limit $\lim _{\leftarrow} C_{2}\left(X_{\alpha}\right)$.

Lemma 1. $\lim p_{\alpha N_{*}}: C_{2}\left(X_{N}\right) \rightarrow \lim C_{2}\left(X_{\alpha}\right)$ is injective and yields an injection from $H_{2}\left(X_{N}\right)$ to $\lim H_{2}\left(X_{\alpha}\right)$ when restricted to $H_{2}\left(X_{N}\right)$; in particular, if all the $H_{2}\left(X_{\alpha}\right)$ are trivial, then $\mathrm{H}_{2}\left(X_{N}\right)$ is trivial.

Proof. First we show that if $c_{1}=N * \tilde{c}_{1}$ and $c_{2}=N * \tilde{c}_{2}$ are two different open cells in $X_{N}$, then there exists an element $\beta \in \Omega$ such that $p_{\beta N}\left(c_{1}\right)$ and $p_{\beta N}\left(c_{2}\right)$ are two different open cells in $X_{\beta}$. Suppose not. Then

$$
G_{\alpha} * \tilde{c}_{1}=G_{\alpha} * \tilde{c}_{2}
$$

for all $\alpha \in \Omega$. So, in particular,

$$
\tilde{c}_{1} \in G_{\alpha} * \tilde{c}_{2}
$$

for all $\alpha \in \Omega$. Then for each $\alpha \in \Omega$ there exists a $g_{\alpha}$ in $G_{\alpha}$ such that

$$
\tilde{c}_{1}=g_{\alpha} * \tilde{c}_{2} \text {. }
$$

Fix an element $\gamma \in \Omega$; then $g_{\alpha} * \tilde{c}_{2}=\tilde{c}_{1}=g_{\gamma} * \tilde{c}_{2}$ for all $\alpha \in \Omega$; hence $g_{\gamma}^{-1} g_{\alpha} * \tilde{c}_{2}=\tilde{c}_{2}$ for all $\alpha \in \Omega$. Since $G$ acts freely on the set of open cells of $\tilde{X}$ this says that $g_{\gamma}^{-1} g_{\alpha}=1$; thus $g_{\gamma}=g_{\alpha} \in G_{\alpha}$ for all $\alpha \in \Omega$ and therefore $g_{\gamma}$ is an element of the intersection $N$. Since

$$
\tilde{c}_{1}=g_{\gamma} * \bar{c}_{2} \text {, }
$$

we have $c_{1}=N * \tilde{c}_{1}=N * \tilde{c}_{2}=c_{2}$, which contradicts our assumption that $c_{1}$ and $c_{2}$ are different cells. Suppose now that

$$
z=\sum_{k=1}^{m} n_{k} c_{k}
$$

is a nontrivial element of $C_{2}\left(X_{N}\right)$, so that the integers $n_{k}$ are nonzero and the cells $c_{k}$ are different 2-cells of $X_{N}$. If $m=1$, then

$$
p_{\alpha N_{*}}(z)=n_{1} p_{\alpha N}\left(c_{1}\right) \neq 0
$$

for all $\alpha \in \Omega$. If $m>1$ then for every pair $\{i, j\}, i, j \in\{1, \ldots, m\}$, we can find an element $\beta(i, j) \in \Omega$ such that $p_{\beta(i, j) N}\left(c_{i}\right)$ and $p_{\beta(i, j) N}\left(c_{j}\right)$ are two different 2-cells of $X_{\beta(i, j)}$. Let $\beta$ be the largest element among the finitely many $\beta(i, j)$. Then $p_{\beta N}\left(c_{i}\right)$ and $p_{\beta N}\left(c_{j}\right)$ are different cells for any pair $(i, j), i, j \in\{1, \ldots, m\}$, so

$$
p_{\beta N_{*}}(z)=\sum_{k=1}^{m} n_{k} p_{\beta N}\left(c_{k}\right) \neq 0 .
$$

This shows that

$$
\lim _{\leftarrow} p_{\alpha N_{*}}(z) \neq 0
$$

LEMMA 2. $\left(\underset{\lim }{\leftarrow} p_{\alpha N_{*}}\right) \circ h_{N}=\underset{\lim }{\longleftarrow} h_{\alpha}$. 
Proof. From the commutative diagram

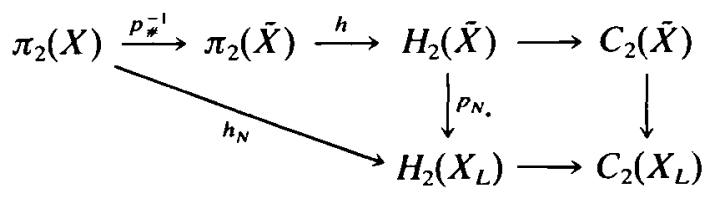

we see that for every $\alpha \in \Omega$,

$$
p_{a N_{*}} \circ h_{N}=p_{\alpha N_{*}}{ }^{\circ} p_{N_{*}} \circ h^{\circ} p_{\#}^{-1}=p_{\alpha_{*}} \circ h^{\circ} p_{\#}^{-1}=h_{\alpha} .
$$

Hence $\left(\lim _{\leftarrow} p_{\alpha N_{*}}\right) \circ h_{N}=\lim _{\longleftarrow} h_{\alpha}$

Proof of Theorem 1. Since $X$ is $G_{\alpha}$-Cockcroft for every $\alpha \in \Omega$, each $h_{\alpha}$ is the zero map. Hence $\lim h_{\alpha}$ is the zero map. Lemma 2 and the fact that, by Lemma $1, \lim p_{\alpha N_{*}}$ is injective show that $h_{N}$ is the zero map as well. So $X$ is $N$-Cockcroft.

Proof of Theorem 2. Only the direction (i) $\Rightarrow$ (ii) requires a proof. If $\left\{G_{\alpha}: \alpha \in \Omega\right\}$ is a chain of subgroups of $G$ such that $H_{2}\left(X_{\alpha}\right)=0$ for all $\alpha$, then $H_{2}\left(X_{N}\right)=0$ by Lemma 1 ; as before $X_{\alpha}$ is the 2-complex $\bar{X}_{G_{\alpha}}$ and $N$ is the intersection of all the $G_{\alpha}$. The existence of a minimal subgroup $L$ such that $H_{2}\left(X_{L}\right)=0$ now follows from Zorn's Lemma. If $L / K$ were a non-trivial $\mathbb{Z}$-conservative quotient of $L$, then $K$ would be a proper subgroup of $L$ with $H_{2}\left(X_{K}\right)=0$ by definition of $\mathbb{Z}$-conservative. This contradicts minimality of $L$.

\section{REFERENCES}

1. J. F. Adams, A new proof of a theorem of W. H. Cockcroft, J. London Math. Soc. 30 (1955), 482-488.

2. K. S. Brown, Cohomology of groups (Springer, 1982).

3. W. A. Bogley, Unions of Cockcroft two-complexes, preprint.

4. W. H. Cockcroft, On two-dimensional aspherical complexes, Proc. London Math. Soc. (3) 4 (1954), 375-384.

5. M. N. Dyer, Cockcroft 2-complexes, preprint. 549-558.

6. J. Howie, Aspherical and acyclic 2-complexes, J. London Math. Soc. (2) 20 (1979),

7. J. Howie, On the fundamental group of an almost-acyclic 2-complex, Proc. Edinburgh Math. Soc. (2) 24 (1981), 119-122.

8. J. Howie, How to generalize one-relator group theory, Combinatorial group theory and topology, ed. S. M. Gersten and J. R. Stallings (Ann. of Math. Stud. 111, Princeton Univ. Press, 1987), 53-78. $409-428$.

9. J. H. C. Whitehead, On adding relations to homotopy groups, Ann. of Math. (2) 42 (1941),

Fachbereich Mathematik

Johann Wolfgang Goethe-Universität

6000 Frankfurt/Main 11 